\title{
Validation of a Hybrid Microwave-Optical Monitor to investigate Thermal Provocation in the Microvasculature
}

\author{
Allann Al-Armaghany ${ }^{1}$, Kenneth Tong ${ }^{1}$, David Highton ${ }^{2}$ and Terence $S$. \\ Leung $^{3}$ \\ ${ }^{1}$ Department of Electronic and Electrical Engineering, University College London, UK \\ ${ }^{2}$ Neurocritical Care, University College London Hospitals, Queen Square, London, UK \\ ${ }^{3}$ Department of Medical Physics \& Biomedical Engineering, University College London, UK
}

\begin{abstract}
We have previously developed a hybrid microwave-optical system to monitor microvascular changes in response to thermal provocation in muscle. The hybrid probe is capable of inducing deep heat from the skin surface using mild microwaves (1-3W) and raises the tissue temperature by a few degrees Celsius. This causes vasodilation and the subsequent increase in blood volume is detected by the hybrid probe using near infrared spectroscopy. The hybrid probe is also equipped with a skin cooling system which lowers the skin temperature while allowing microwaves to warm up deeper tissues. The hybrid system can be used to assess the condition of the vasculature in response to thermal stimulation. In this validation study, thermal imaging has been used to assess the temperature distribution on the surface of phantoms and human calf, following microwave warming. The results show that the hybrid system is capable of changing the skin temperature with a combination of microwave warming and skin cooling. It can also detect thermal responses in terms of changes of oxy/deoxy-hemoglobin concentrations.
\end{abstract}

\section{Introduction}

Thermal provocation has been used in combination with skin blood flow measurement to assess a number of conditions including chronic spinal cord injury [1], digital obstructive arterial disease (DOAD) [2] and transplanted free flaps [3]. In these studies, the region of interest is warmed by placing it into a thermal box [2], immersing it into warm water [3] or using a heating element directly on the skin [1], and the subsequent changes in blood flow are measured by a laser Doppler flowmeter. Healthy skin will respond by vasodilation leading to an increase in blood flow, while suboptimal or pathological skin may initiate a much smaller increase. It is believed that there are at least two factors which may affect the magni- 
tude of the thermal response: (1) the obstruction of the vasculature as in the case of DOAD [2], and (2) the disruption of the sympathetic pathways as in the case of spinal cord injury [1] and transplanted free flaps [3].

Although current studies focus on the skin, one natural progression of this research is to investigate the thermal response of the muscle below the skin and see whether similar observations can be found. This work aims to develop a device to facilitate such investigations. In our previous publication, we described the development of a hybrid microwave-optical system to monitor thermal provocation in muscle [4]. The hybrid probe has integrated a number of devices including (i) a microwave patch applicator to induce deep heat, (ii) a NIRS (near infrared spectroscopy) optical probe to monitor changes in oxy/deoxy/total-hemoglobin concentration $\left(\Delta\left[\mathrm{HbO}_{2}\right] / \Delta[\mathrm{HHb}] / \Delta[\mathrm{HbT}]\right)$ in the tissue, (iii) a temperature sensor to monitor skin temperature, (iv) a skin cooling system to minimize skin temperature, and (v) a laser Doppler probe to monitor skin blood flow. Since the NIRS and laser Doppler probes interfere with each other in the current design, they cannot be used simultaneously. The whole system has been built in-house.

In this paper, thermal imaging has been adopted to investigate the temperature distribution in phantoms and human calf, following microwave warming, with and without skin cooling. The hybrid probe remains largely the same as the previous design and its details can be found in the previous publication [4]. The current design has a small modification on the location of the temperature sensor to improve the feedback on skin cooling.

\section{Methods}

\subsection{Phantom Study}

A cylindrical tissue mimicking phantom was made which consisted of three layers (thicknesses): skin $(2 \mathrm{~mm})$, fat $(10 \mathrm{~mm})$ and muscle $(40 \mathrm{~mm})$ layers, with a diameter of $120 \mathrm{~mm}$. The ingredients for the phantom included deionized water, gelatin, paraffin, safflower oil, detergent, n-propanol, formaldehyde and p-tolic acid [5]. The proportion of the ingredients was adjusted for the three layers separately so that their dielectric properties matched those found in real human tissues. The relative permittivity/electrical conductivity $(\mathrm{S} / \mathrm{m})$ were measured to be 37.7/1.6, 5.6/0.2 and 54.7/2.2 for the skin, fat and muscle layers, respectively.

To establish the amount and depth of heat that the hybrid probe can induce, it was placed on top of the phantom and $3 \mathrm{~W}$ microwaves were used to warm up the phantom. After $5 \mathrm{~min}$, the microwave system was switched off and the hybrid probe was removed to expose the skin phantom's surface so that a thermal image can be taken with an infrared thermal camera (FLIR i7). Afterwards, the skin and 
fat phantom layers were also removed to expose the top of the fat phantom layer for thermal images to be taken. The experiment was conducted twice, one with skin cooling and one without.

\subsection{In vivo Study}

The in vivo study has been approved by the UCL Ethics Committee. Three adult subjects were involved in this study. The subject sat in a comfortable chair in a quiet room. The hybrid probe was placed securely on the calf of the subject's leg. The placement of the hybrid probe was judged satisfactory if the corresponding NIRS signals $\left(\Delta\left[\mathrm{HbO}_{2}\right] / \Delta[\mathrm{HHb}]\right)$ had good signal quality. The skin temperature and the NIRS signals typically settled to a stable level after approximately $10 \mathrm{~min}$. The monitoring then started in the resting state, lasting for 5 to $7 \mathrm{~min}$, after which the microwave system was switched on for a period of time. Afterwards, the monitoring continued for another 20 min during the recovery state.

To establish how the combination of microwave warming and skin cooling affected the results, a series of experiments were conducted for three cases, i.e., case I: microwave warming without skin cooling, case II: microwave warming with skin over cooling, and case III: microwave warming with moderate skin cooling. In case I, $1 \mathrm{~W}$ microwaves were used for $60 \mathrm{~min}$ without skin cooling. In case II, 1 $\mathrm{W}$ microwaves were used for $60 \mathrm{~min}$, this time with skin cooling to $1^{\circ} \mathrm{C}$ below the initial temperature (over cooling). In case III, $3 \mathrm{~W}$ microwaves were used for 15 min, with skin cooling to $0.2^{\circ} \mathrm{C}$ below the initial temperature. In cases I, II and III, the experiments were repeated again but this time the hybrid probe was removed immediately after the microwave system was switched off to allow thermal images to be taken.

\section{$3 \quad$ Results}

\subsection{Phantom results}

Figure 1(a) and (b) show the thermal images taken on the skin and muscle phantoms after 5 min of microwave warming without skin cooling. It can be seen that for the skin phantom, the temperature at the central cursor location was raised to $28.6^{\circ} \mathrm{C}$ (from $20^{\circ} \mathrm{C}$ ) while the temperature of the muscle phantom raised to $25.1^{\circ} \mathrm{C}$, confirming the capability of the microwave applicator to induce deep heat. With the skin cooling system switched on, the skin phantom temperature only raised slightly to $21.5^{\circ} \mathrm{C}$ (from $19^{\circ} \mathrm{C}$ ) as shown in Fig.1(c). The muscle phan- 
tom temperature, however, was raised to $24.4^{\circ} \mathrm{C}$ as depicted in Fig.1(d), confirming the capability of the skin cooling system to reduce superficial temperature and that of the microwave applicator to induce deep heat.

\subsection{In vivo results}

Figure 2(a) and (b) show case I (microwave warming without skin cooling) results including the $\Delta\left[\mathrm{HbO}_{2}\right] / \Delta[\mathrm{HHb}] / \Delta[\mathrm{HbT}]$ signals and the skin temperature during the experiment. Figure 2(c) depicts the thermal image of the skin surface immediately after $60 \mathrm{~min}$ of microwave warming in a second experiment. It can be seen that the skin temperature increased rapidly at the beginning and then settled at $34.7^{\circ} \mathrm{C}$ approximately $24 \mathrm{~min}$ into microwave warming. In the meantime, both $\Delta\left[\mathrm{HbO}_{2}\right]$ and $\Delta[\mathrm{HbT}]$ increased significantly, showing that the blood volume increase was mainly due to the dilation of arterial vessels, while the slight decrease in $\Delta[\mathrm{HHb}]$ indicated an increased blood flow.

Figure 3 shows case II (microwave warming with skin over cooling) results. The skin cooling system lowered the skin temperature to $\sim 1^{\circ} \mathrm{C}$ below the initial temperature, as shown in Fig.3(b). The corresponding $\Delta\left[\mathrm{HbO}_{2}\right]$ and $\Delta[\mathrm{HbT}]$ in Fig. 3 decreased gradually while $\Delta[\mathrm{HHb}]$ remained relatively stable. These behaviors are consistent with vasoconstriction in the arterial vessels which is what one would expect when human tissue is cooled. The thermal image in Fig. 3(c) also confirms the lowering of skin temperature. It is noted from Fig. 3(b) that when both the microwave and skin cooling systems were switched off, the skin temperature rose significantly before it gradually went down again, possibly due to a combination of rewarming of the skin and the diffusion of heat from the microwave applicator back to the skin.

Figure 4 shows case III (microwave warming with moderate skin cooling) results. The microwave power was $3 \mathrm{~W}$ higher than those used in the previous two cases. The warming duration was therefore shortened to $15 \mathrm{~min}$ to avoid over warming the muscle. Figure 4(b) shows that the skin cooling system kept the skin temperature at $\sim 0.2^{\circ} \mathrm{C}$ below the initial temperature. Figure 4(a) shows that after the microwaves were turned on, $\Delta\left[\mathrm{HbO}_{2}\right]$ and $\Delta[\mathrm{HbT}]$ initially decreased for approximately $3 \mathrm{~min}$, followed by an increase in $\Delta\left[\mathrm{HbO}_{2}\right]$ and a decrease in $\Delta[\mathrm{HHb}]$ over the next $12 \mathrm{~min}$. In comparison to case I, the increase of $\Delta\left[\mathrm{HbO}_{2}\right]$ in case III was much smaller. After the microwaves were switched off, the skin temperature increased momentarily and then dropped, as in the case of case II. As for $\Delta\left[\mathrm{HbO}_{2}\right]$, this gradually decreased back to the initial level while $\Delta[\mathrm{HHb}]$ continued to decrease. It is noted that although the skin temperature was kept at below the initial temperature of $30.5^{\circ} \mathrm{C}$ as shown in Fig. 4(b), the thermal image in Fig. 4(c) shows that the temperature distribution on the skin was not evenly distributed. The skin temperature near the optical probe had in fact increased, causing an increase in $\Delta\left[\mathrm{HbO}_{2}\right]$ and a decrease in $\Delta[\mathrm{HHb}]$ on the skin. 


\section{Discussions and Conclusions}

This study has shown that by combing microwave deep warming and skin cooling, the skin temperature can be adjusted accordingly. In order to investigate the thermal provocation of muscle microvasculature, it would be necessary to be able to warm preferentially the deeper region while maintaining the skin temperature relatively constant so that any changes in the NIRS signals could be related to the muscle rather than to the skin. This preferential warming can potentially be achieved by optimizing the balance between microwave warming and skin cooling. However, the thermal imaging results suggest that the current design of the hybrid probe needs to be modified to achieve this. The main technical problem, as revealed by the thermal images, is that the skin temperature distribution is not uniform and the skin temperature indicated by the temperature sensor is only valid for its immediate neighboring region. As a result, the skin cooling only aims to maintain the skin temperature at a certain level for a small area under the temperature sensor, rather than for the whole region under the hybrid probe. To tackle this problem, a more thorough and representative temperature monitoring system needs to be introduced and a more effective configuration of cooling the skin at the right place would also be necessary. The initial aim of this work is to develop a device which facilitates investigations into thermal provocation in deep tissues. As for the accompanied physiology, it will be left to be investigated in the next phase of this work in a larger scale study involving many more subjects.

Acknowledgement This work was partly funded by EPSRC (Grant Code EP/G005036/1).

\section{References}

[1] A. Nicotra, M. Asahina, and C. J. Mathias, "Skin vasodilator response to local heating in human chronic spinal cord injury," European Journal of Neurology, vol. 11, pp. 835-837, // 2004.

[2] G. Mahe, D. A. Liedl, C. McCarter, R. Shepherd, P. Gloviczki, I. R. McPhail, et al., "Digital obstructive arterial disease can be detected by laser Doppler measurements with high sensitivity and specificity," Journal of Vascular Surgery, vol. 59, pp. 1051-1057.e1, 2014.

[3] A. Rahmanian-Schwarz, J. L. Schiefer, A. Amr, J. Rothenberger, H. E. Schaller, and B. Hirt, "Thermoregulatory response of anterolateral thigh flap compared with latissimus dorsi myocutaneous flap: an evaluation of flaps cutaneous flow and velocity due to thermal stress," Microsurgery, vol. 31, pp. 650-4, Nov 2011.

[4] A. Al-Armaghany, K. Tong, and T. S. Leung, "Development of a hybrid microwave-optical thermoregulation monitor for the muscle," Adv Exp Med Biol, vol. 812, pp. 347-53, 2014.

[5] M. Lazebnik, E. L. Madsen, G. R. Frank, and S. C. Hagness, "Tissue-mimicking phantom materials for narrowband and ultrawideband microwave applications," Physics in Medicine and Biology, vol. 50, pp. 4245-4258, 2005. 

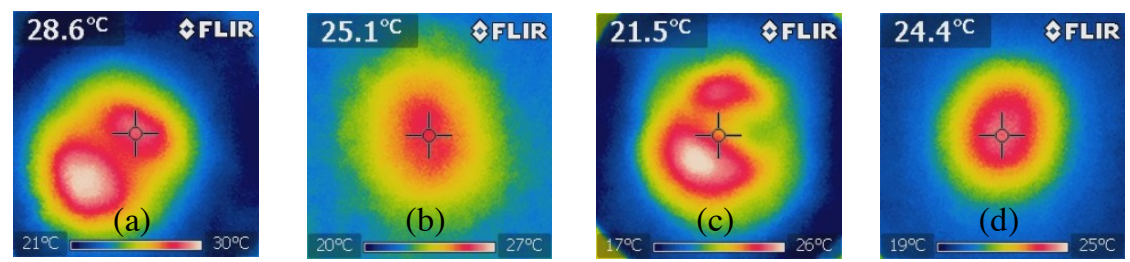

Fig. 1 Thermal images of phantom during microwave exposure (a) skin phantom without cooling, (b) muscle phantom without cooling (c) skin phantom with cooling (d) muscle phantom with cooling
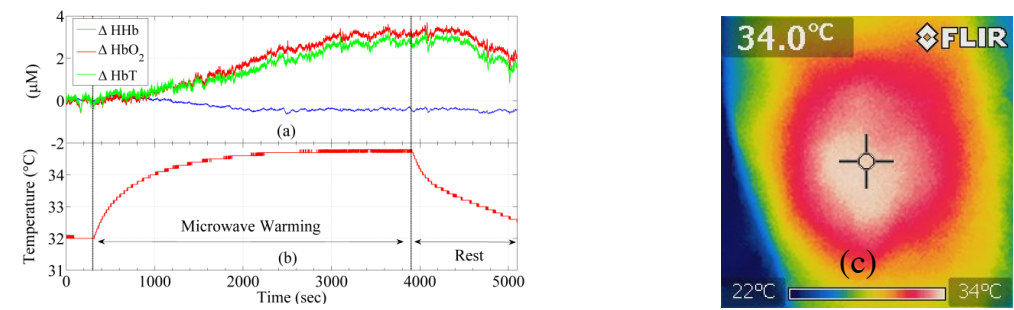

Fig. 2 Case I: with microwave warming without cooling (a) Measured NIRS signals, (b) Measured skin temperature (c) Skin thermal image immediately after warming.
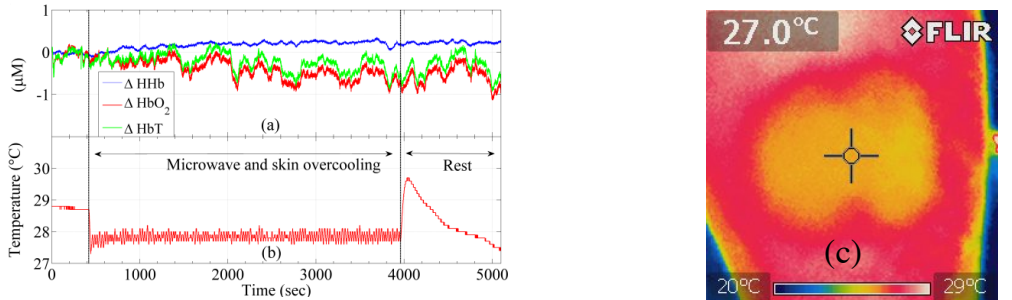

Fig. 3 Case II: microwave warming with skin over cooling $\left(1{ }^{\circ} \mathrm{C}\right.$ below initial temperature $)$ (a) Measured NIRS signals, (b) Measured skin temperature (c) Skin thermal image
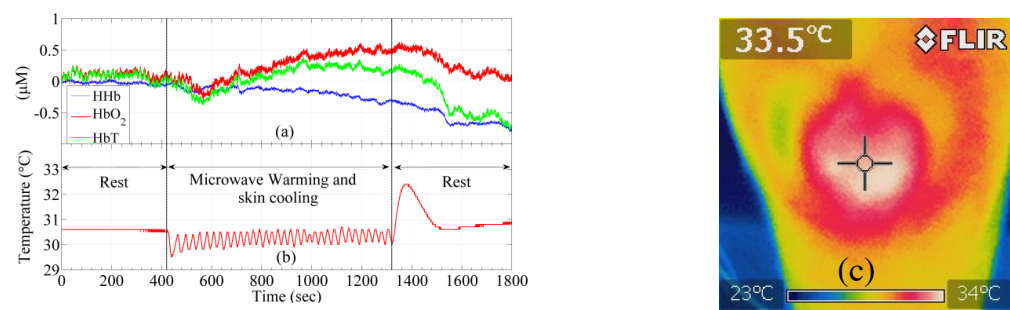

Fig. 4 Case III: microwave warming at $3 \mathrm{~W}$ with skin moderate cooling $\left(0.2^{\circ} \mathrm{C}\right.$ below initial temperature) (a) Measured NIRS signals, (b) Measured skin temperature (c) Skin thermal image 